\title{
Ductal Breast Carcinoma In Situ
}

National Cancer Institute

\section{Source}

National Cancer Institute. Ductal Breast Carcinoma In Situ. NCI Thesaurus. Code C2924.

A carcinoma entirely confined to the mammary ducts. It is also known as DCIS. There is no evidence of invasion of the basement membrane. Currently, it is classified into three categories: High-grade DCIS, intermediate-grade DCIS and low-grade DCIS. In this classification the DCIS grade is defined by a combination of nuclear grade, architectural growth pattern and presence of necrosis. The size of the lesion as well as the grade and the clearance margins play a major role in dictating the most appropriate therapy for DCIS. 